\title{
Locus heterogeneity
}

National Cancer Institute ( $\mathrm{NCl})$

\section{Source}

National Cancer Institute (NCI). Locus heterogeneity.

The same phenotype is caused by mutations in genes at different chromosomal loci. 